\title{
NONLINEAR HARMONIC GENERATION AND PROPOSED EXPERIMENTAL VERIFICATION IN SASE FELS*
}

\author{
H.P. Freund \\ Science Applications International Corporation \\ McLean, VA 22102 \\ S.G. Biedron and S.V. Milton \\ Advanced Photon Source, Argonne National Laboratory \\ Argonne, IL 60439
}

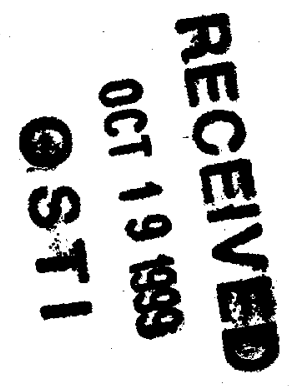

\begin{abstract}
Recently, a 3D, polychromatic, nonlinear simulation code was developed to study the growth of nonlinear harmonics in self-amplified spontaneous emission (SASE) freeelectron lasers (FELs). The simulation was applied to the parameters for each stage of the Advanced Photon Source (APS) SASE FEL, intended for operation in the visible, UV, and short UV wavelength regimes, respectively, to study the presence of nonlinear harmonic generation. Significant nonlinear harmonic growth is seen. Here, a discussion of the code development, the APS SASE FEL, the simulations and results, and, finally, the proposed experimental procedure for verification of such nonlinear harmonic generation at the APS SASE FEL will be given.
\end{abstract}

*Work supported by the United States Department of Energy, Office of Basic Energy Sciences, under Contract No. W-31-109-ENG-38.

The submitted manuscript has been created by the University of Chicago as Operator of Argonne National Laboratory ("Argonne") under Contract No. W-31-109-ENG-38 with the U.S. Department of Energy. The U.S. Government retains for itself, and others acting on its behalf, a paid-up, nonexclusive, irrevocable worldwide license in said article to reproduce, prepare derivative works, distribute copies to the public, and perform publicly and display publicly, by or on behalf of the Government. 


\section{DISCLAIMER}

This report was prepared as an account of work sponsored by an agency of the United States Government. Neither the United States Government nor any agency thereof, nor any of their employees, make any warranty, express or implied, or assumes any legal liability or responsibility for the accuracy, completeness, or usefulness of any information, apparatus, product, or process disclosed, or represents that its use would not infringe privately owned rights. Reference herein to any specific commercial product, process, or service by trade name, trademark, manufacturer, or otherwise does not necessarily constitute or imply its endorsement, recommendation, or favoring by the United States Government or any agency thereof. The views and opinions of authors expressed herein do not necessarily state or reflect those of the United States Government or any agency thereof. 


\section{DISCLAIMER}

Portions of this document may be illegible in electronic image products. Images are produced from the best available original document. 


\section{Introduction}

An important goal of the synchrotron radiation community is to reach shorter wavelengths than $1 \AA$ with higher coherent powers. There are several techniques under consideration to reach these goals: SASE (self-amplified spontaneous emission) at shorter wavelengths [1], which requires higher beam energies and/or shorter wiggler periods; the two-wiggler FEL amplifier scheme [2]; and high-gain harmonic generation [3]. In SASE FELs, there are naturally occuring nonlinear harmonics that could be used to reach these shorter, desired wavelengths. In this paper, a discussion of the nonlinear mechanism is provided. In addition, the application of the 3D polychromatic code MEDUSA to several planned experiments on the SASE FEL under construction at the Advanced Photon Source (APS) is described.

\section{The Multi-Purpose Simulation Code: MEDUSA}

MEDUSA is based on a Gauss-Hermite mode expansion [4], in conjunction with a source-dependent expansion [5] for the dynamic evolution of the mode spot sizes and the curvature of the phase fronts. As a result, MEDUSA constitutes a slow-time-scale formulation of Maxwell's equations where the amplitudes and phases of each mode, as well as the spot size and phase front curvature of each wavelength component, are integrated in conjunction with the 3D Lorentz force equations for an ensemble of electrons. Note that no wiggler averaging is imposed on the Lorentz force equations. Either parabolic-pole-face or flat-pole-face undulator models may be employed in singleor multiple-segmented configuration. Quadrupoles are also available for matching conditions between undulator segments, and dipoles may be used to simulate spurious 
kicks and/or dispersive sections. Finally, either Gaussian or Waterbag electron beam distributions may be used.

MEDUSA has been benchmarked in monochromatic mode with four other simulation codes [6] and to the linear theory [7], and good agreement has been found among all of the formulations.

More recently, MEDUSA has been extended to include multiple frequencies [8]. In a monochromatic slow-time-scale formulation (with angular frequency $\omega$ ), Maxwell's equations are averaged over the wave period $2 \pi / \omega$. A polychromatic formulation is obtained by the inclusion of an arbitrary ensemble of Gauss-Hermite modes where the frequencies were all multiples of some $\Delta \omega$. In this representation, Maxwell's equations are averaged over a period $2 \pi / \Delta \omega$. This technique is quite general and can be used to treat harmonic interactions and/or sidebands. In the treatment of sidebands, $\Delta \omega<<\omega$ and the averaging period is long in comparison to that used for a monochromatic simulation. This requires the use of a large number of electrons in simulation. In contrast, harmonics are treated using $\Delta \omega=\omega$, however, more electrons are also required to resolve the harmonic variation in phase space. As a result, both cases result in typically longer run times than a monochromatic simulation. In this paper we are concerned with the harmonic interactions.

\section{The Simulation Subject: The APS SASE FEL}

A SASE FEL with an initial wavelength of $532 \mathrm{~nm}$ is currently being commissioned at the Advanced Photon Source at Argonne National Laboratory [9]. This project will be performed in three stages as summarized in Table 1. The APS SASE FEL is composed of a drive-laser system [10] for a photocathode if gun [11] or a thermionic rf 
gun [12], a 700-MeV electron linac [13] and two transfer lines, an undulator hall, a diagnostics end station, and numerous electron and light beam diagnostics [14-16]. A segmented undulator is used. Each unit cell is $2.7 \mathrm{~m}$ in length and composed of a $2.4-\mathrm{m}$ undulator, $0.3 \mathrm{~m}$ of electron and photon diagnostics, and a combined function quadrupole and corrector magnet [17]. The undulator parameters are listed in Table 2.

Table 1: Regimes of Operation

\begin{tabular}{|l|c|c|c|}
\hline Parameter & Stage I & Stage II & Stage III \\
\hline Beam Energy $(\mathrm{MeV})$ & 216 & 456 & 707 \\
\hline Wavelength $(\mathrm{nm})$ & 532 & 120 & 50 \\
\hline Emittance $(\pi \mathrm{mm}-\mathrm{mrad})$ & 5 & 3 & 3 \\
\hline Energy Spread $(\%)$ & $0.1 \%$ & $0.1 \%$ & $0.1 \%$ \\
\hline Peak Current $(\mathrm{A})$ & 150 & 300 & 500 \\
\hline Schedule & 1999 & 2000 & 2001 \\
\hline
\end{tabular}

Table 2: Undulator Parameters

\begin{tabular}{|l|c|}
\hline \multicolumn{1}{|c|}{ Parameter } & Value \\
\hline On-axis Undulator Strength $(\mathrm{kG})$ & 10.61 \\
\hline Length $(\mathrm{m})$ & 2.4 \\
\hline Undulator Period $(\mathrm{cm})$ & 3.3 \\
\hline $\mathrm{K}$ & 3.1 \\
\hline
\end{tabular}

\section{Simulation Results}

Simulations were performed including nine harmonics for each of the three APS SASE FEL stages. MEDUSA was first used to find the energy yielding the optimum gain at the desired fundamental wavelengths $(532 \mathrm{~nm}, 120 \mathrm{~nm}$, and $50 \mathrm{~nm})$. These are the beam energies shown in Table 1. A single-segment parabolic-pole-face undulator is treated in this paper since the basic physics of the nonlinear harmonic interaction is more easily presented using this simpler undulator geometry. In all the simulations described herein, a 10-W signal was injected at the fundamental. Higher harmonics are undriven and grow parasitically off the fundamental as the electron beam becomes bunched. 
The growth of the fundamental and the odd and even harmonics for Stage I are shown in Figs. 1 and 2, respectively. The fundamental grows exponentially with a gain length of $0.5925 \mathrm{~m}$ in close agreement with the linear theory [7]. Although the harmonics are undriven, they grow parasitically off bunching at the fundamental, and it is important to observe that the harmonic growth rates are faster than the fundamental growth rate. Indeed, the gain length scales inversely with the harmonic number so that the gain length at the $h^{\text {th }}$ harmonic scales approximately as $1 / h$ times the fundamental gain length.

In order to illustrate this scaling, consider Maxwell's equation in 1-D,

$$
\left(\frac{\partial^{2}}{\partial z^{2}}-\frac{1}{c^{2}} \frac{\partial^{2}}{\partial t^{2}}\right) E_{x}(z, t)=\frac{4 \pi}{c^{2}} \frac{\partial}{\partial t} J_{x}(z, t)
$$

where the electric field is given by

$$
E_{x}(z, t)=\frac{1}{2} \sum_{h} \hat{E}_{h}(z) \exp \left[i h k_{0}(z-c t)\right]+c . c .,
$$

and the source current is given in terms of a nonlinear conductivity, $\sigma_{h}^{(N L)}$,

$$
J_{x}=\frac{1}{2} \sum_{h}\left[\sigma_{h}^{(L)} \hat{E}_{h}+\sigma_{h}^{(N L)} \hat{E}_{1}^{h}\right] \exp \left[i h k_{0}(z-c t)\right]+c . c .,
$$

where $\sigma_{h}^{(L)}$ describes the linear interaction. It is not important to specify the detailed form for the nonlinear conductivity here other than to note that it is included implicitly in the nonlinear formulation. These nonlinear terms are driven by bunching at the fundamental, which dominates the interaction, and we can write Maxwell's equations as

$$
\frac{d}{d z} \hat{E}_{h} \cong-\frac{2 \pi}{c}\left(\sigma_{h}^{(L)} \hat{E}_{h}+\sigma_{h}^{(N L)} \hat{E}_{1}^{h}\right)
$$

Therefore, if the fundamental grows as $\hat{E}_{1} \approx \exp [\Gamma z]$ then the harmonics will grow as $\hat{E}_{1}^{h} \approx \exp [h \Gamma z]$, and the gain length scales as $L_{g} \approx(2 h \Gamma)^{-1}$. The scaling of the gain 
length with the harmonic number is characteristic of the nonlinear mechanism. It is a well-known phenomenon in Traveling Wave Tubes [18] and was also seen in a 1D analysis for the first and third harmonics in an FEL [19]. More recently, the present analysis has motivated a 3D analytical model that is under development [20].

The evolution of the fundamental and the odd and even harmonics for Stages II and III are shown in Figs. 3-6, and a summary of the gain lengths and saturated powers for all three stages is given in Table 3. The $1 / h$ scaling of the gain length is observed in each of these regimes, and the powers in the harmonics can be substantial. Note that the harmonic powers given in Table 3 refer to the peak powers found at the harmonics over the interaction lengths studied, and not the harmonic powers at the saturation point for the fundamental. This choice was made because the harmonic evolution differs from the fundamental, and the harmonic powers peak at different positions. Thus, a different choice for the overall interaction length would be made for a specific harmonic. Note also that while the even harmonic gain lengths exhibit the $1 / h$ scaling, the even harmonic powers are much lower than those at the adjacent odd harmonics. This is due to the planar undulator geometry, which favors the odd harmonics. 
Table 3: Gain Lengths and Saturated Powers

\begin{tabular}{|l|l|l|l|l|l|l|}
\hline $\boldsymbol{h}$ & \multicolumn{2}{|c|}{ Stage I } & \multicolumn{2}{c|}{ Stage II } & \multicolumn{2}{c|}{ Stage III } \\
\hline & $L_{G}(\mathrm{~m})$ & $P_{\text {sat }}$ & $L_{G}(\mathrm{~m})$ & $P_{\text {sat }}$ & $L_{G}(\mathrm{~m})$ & $P_{\text {sat }}$ \\
\hline 1 & 0.5925 & $138.4 \mathrm{MW}$ & 1.039 & $402.2 \mathrm{MW}$ & 1.418 & $449.9 \mathrm{MW}$ \\
\hline 2 & 0.3460 & $3.276 \mathrm{~kW}$ & 0.5964 & $15.92 \mathrm{~kW}$ & 0.6979 & $28.83 \mathrm{~kW}$ \\
\hline 3 & 0.2062 & $832.6 \mathrm{~kW}$ & 0.4113 & $2.461 \mathrm{MW}$ & 0.4768 & $1.509 \mathrm{MW}$ \\
\hline 4 & 0.1610 & $1.365 \mathrm{~kW}$ & 0.2784 & $803.2 \mathrm{~W}$ & 0.2639 & $10.39 \mathrm{~kW}$ \\
\hline 5 & 0.1248 & $42.04 \mathrm{~kW}$ & 0.2643 & $724.2 \mathrm{~kW}$ & 0.2690 & $1.489 \mathrm{MW}$ \\
\hline 6 & 0.1015 & $381.8 \mathrm{~W}$ & 0.1888 & $2.948 \mathrm{~kW}$ & 0.2351 & $5.887 \mathrm{~kW}$ \\
\hline 7 & 0.0856 & $39.39 \mathrm{~kW}$ & 0.1784 & $38.90 \mathrm{~kW}$ & 0.2203 & $323.5 \mathrm{~kW}$ \\
\hline 8 & 0.0872 & 207.4 & 0.1442 & $973.6 \mathrm{~W}$ & 0.1970 & $319.6 \mathrm{~W}$ \\
\hline 9 & 0.0670 & $39.99 \mathrm{~kW}$ & 0.1324 & $13.98 \mathrm{~kW}$ & 0.1614 & $49.13 \mathrm{~kW}$ \\
\hline
\end{tabular}

\section{Plan for Experimental Verification}

We plan to perform experiments to examine the first through third harmonics and verify the nonlinear growth, in multiple-shot and single-shot modes [21]. Since the third harmonic of $532 \mathrm{~nm}$ is already into the vacuum ultraviolet, we intend to lower the electron beam energy and retune the linac and electron beam quality to provide SASE at a longer wavelength. The precise wavelength will be determined after commissioning is complete at $532 \mathrm{~nm}(\sim 217 \mathrm{MeV})$, since the linac and transfer lines have to be reoptimized. We will attempt to place the first harmonic as low as the electron beam energy and corresponding performance will allow. There currently exists an optical transfer line to transmit the SASE light to the diagnostics end station.

The first technique will consist of two filter wheels and a detector. The first, sixposition filter wheel is to house three "pairs" of bandpass filters. In the first position, the filter will pass the spontaneous radiation of the first harmonic and the second position will pass the SASE of the first harmonic. The other two sets will pass the spontaneous and SASE for the second and third harmonics, respectively. 
The second filter wheel will house calibrated neutral density filters to control the light intensity at the detector. The detector type is a UV-enhanced silicon photodiode, calibrated in the wavelength region of $180-1100 \mathrm{~nm}$. The absolute and relative intensity level of each harmonic thus obtained will be compared with theory and simulation using the above-mentioned neutral density filters.

The single-shot method will employ a commercially available grating polychromator capable of simultaneously resolving up to the first three harmonics. Three identical silicon photodiode detectors will be used with appropriate neutral density filters for balancing the intensity variations between harmonics.

\section{Conclusions}

In summary, the 3D simulation code MEDUSA has been extended to simulate harmonic interactions in FELs. Simulations and analyses for the three stages of the APS SASE FEL indicate strong nonlinear harmonic generation can be expected. We conclude from the present study that significant nonlinear harmonic components will be present in SASE FELs that will be important in extending the wavelength region to shorter wavelengths. In addition to this work, an analytical model is being developed.

Currently, two types of experiments are planned to measure the nonlinear harmonic growth and the scaling of the gain lengths. Further experiments are also under consideration, including measuring the mode structure in the higher harmonics and comparing it to a simulated mode structure, and measuring the coherence length of the harmonics. 


\section{Acknowledgements}

The authors wish to thank the following individuals for their support in preparing this manuscript and the corresponding oral presentation: Z. Huang, K-J. Kim, and L.C. Teng.

\section{References}

[1] S.V. Milton et al., "FEL Development at the APS: The APS SASE FEL," in FreeElectron Laser Challenges II, Harold E. Bennett, David H. Dowell, Editors, Proceedings of SPIE Vol. 3614, 86-95 (1999); The VISA Collaboration (BNL, LANL, LLNL, SLAC, UCLA), VISA Proposal, "Proposal for a SASE-Free-electron Laser Experiment, VISA, at the ATF linac," April 1998; "A VUV Free Electron Laser at the TESLA Test Facility at DESY: Conceptual Design Report," DESY Print, TESLA-FEL 95-03, June 1995; LCLS Design Study Group, Linac Coherent Light Source (LCLS) Design Study Report, SLAC-R-521.

[2] R. Bonifacio, L. De Salvo Souza, P. Pierini, and E.T. Scharlemann, "Generation of XUV Light by Resonant Frequency Tripling in a Two-Wiggler FEL Amplifier," NIM A 296, 787-790 (1990).

[3] L.H. Yu, "Generation of intense uv radiation by subharmonically seeded single-pass free-electron lasers," Phys. Rev. A 44, 5178 (1991).

[4] H.P. Freund, Phys. Rev. E 52, 5401 (1995); S.G. Biedron, H.P. Freund, and S.V. Milton, "3D FEL code for the simulation of a high-gain harmonic generation experiment," in Free-Electron Laser Challenges II, Harold E. Bennett, David H. Dowell, Editors, Proceedings of SPIE Vol. 3614, 96-108 (1999).

[5] P. Sprangle, A. Ting, and C.M. Tang, Phys. Rev. A 36, 2773 (1987). 
[6] S.G. Biedron, Y.C. Chae, R.J. Dejus, B. Faatz, H.P. Freund, S.V. Milton, H-D. Nuhn, S. Reiche, "Multi-Dimensional Free-Electron Laser Simulation Codes: A Comparison Study," these proceedings.

[7] L.H. Yu et al., Phys. Rev. Lett. 64, 3011 (1990); M. Xie, in Proceedings of the IEEE 1995 Particle Accelerator Conference, 183 (1995).

[8] H.P. Freund, S.G. Biedron, and S.V. Milton, IEEE J. Quantum Electronics (submitted for publication).

[9] S.V. Milton, "The low-energy undulator test line," in Free-Electron Laser Challenges, Patrick G. O'Shea, Harold Bennett, Editors, Proceedings of SPIE Vol. 2988, 20-27 (1997).

[10] G. Travish, N. Arnold, and R. Koldenhoven,"The Drive Laser for the LEUTL FEL RF Photoinjector," these proceedings.

[11] S. G. Biedron, G.A. Goeppner, J.W. Lewellen, S.V. Milton, A. Nassiri, G. Travish, X.J. Wang et al, "The Operation of the BNLATF GUN-IV Photocathode RF Gun at the Advanced Photon Source," Proceedings of the 1999 Particle Accelerator Conference, New York, NY, March 29-April 2, 1999 (to be published).

[12] J.W. Lewellen, S. Biedron, A. Lumpkin, S.V. Milton, A. Nassiri, S. Pasky, G. Travish, M. White, "Operation of the APS RF Gun," Proceedings of the XX International Linear Accelerator Conference, Chicago, Illinois, August 23-28, 1998, 863-865 (1999).

[13] M. White, N. Arnold, W. Berg, A. Cours, R. Fuja, A.E. Grelick, K. Ko, Y.L. Qian, T. Russell, N. Sereno, and W. Wesolowski, "Construction, Commissioning, and Operational Experience of the Advanced Photon Source (APS) Linear Accelerator," 
Proceedings of the XVIII International Linear Accelerator Conference, Geneva, Switzerland, 26-30 August, 1996, 315-319 (1996).

[14] A.H. Lumpkin, W.J. Berg, B.X. Yang, "Planned Optical Diagnostics for the APS Low-Energy Undulator Test Line," 1997 Particle Accelerator Conference, 871-873 (1998).

[15] A.J. Gorski, R.M. Lill, "Construction and Measurement Techniques for the APS LEUTL Project RF Beam Position Monitors," 1999 Particle Accelerator Conference, New York, N.Y., March 29-April 2, 1999, to be published.

[16] G. Decker, O. Singh, A. Pietryla, and C. Gold, private communication.

[17] E. Gluskin et al., "The Magnetic and Diagnostics Systems for the Advanced Photon Source Self-Amplified Spontaneously Emitting FEL," in NIM Proceedings of the $20^{\text {th }}$ International FEL Conference, to be published.

[18] J.W. Hansen, G.A. Lange, A.S. Rostad and R.L. Woods, "System aspects of communications TWTAs on how to deal with the tube manufacturer to your best advantage," Hughes Aircraft Company Electron Dynamics Division Applications Note, August 1992.

[19] R. Bonifacio, L. DeSalvo, and P. Pierini, "Large Harmonic Bunching in a HighGain Free-Electron Laser," NIM A 293 627-629 (1990).

[20] Z. Huang and K. Kim, "Three-Dimensional Analysis of Harmonic Generation in Self Amplified Spontaneous Emission," these proceedings.

[21] B. Yang and S.V. Milton, private communication. 


\section{FIGURE CAPTIONS}

Fig. 1 Power versus distance for Stage I for the odd harmonics.

Fig. 2 Power versus distance for Stage I for the even harmonics.

Fig. 3 Power versus distance for Stage II for the odd harmonics.

Fig. 4 Power versus distance for Stage II for the even harmonics.

Fig. 5 Power versus distance for Regime III for the odd harmonics.

Fig. 6 Power versus distance for Regime III for the even harmonics. 


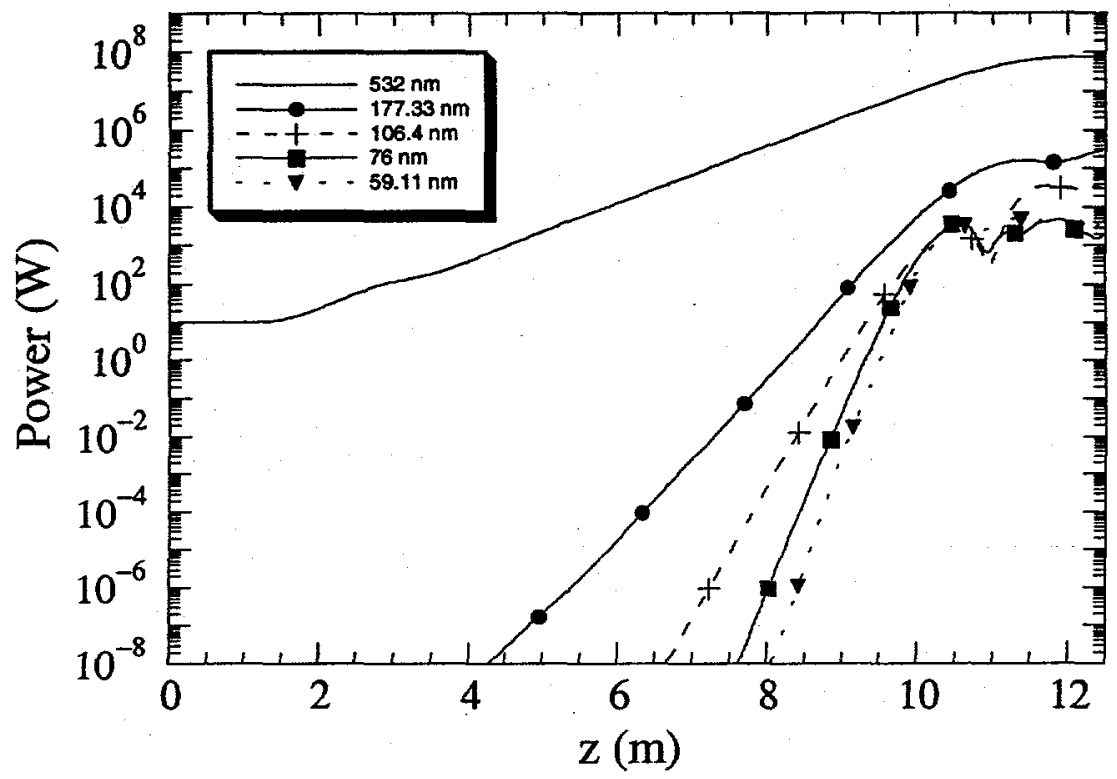

Fig. 1 


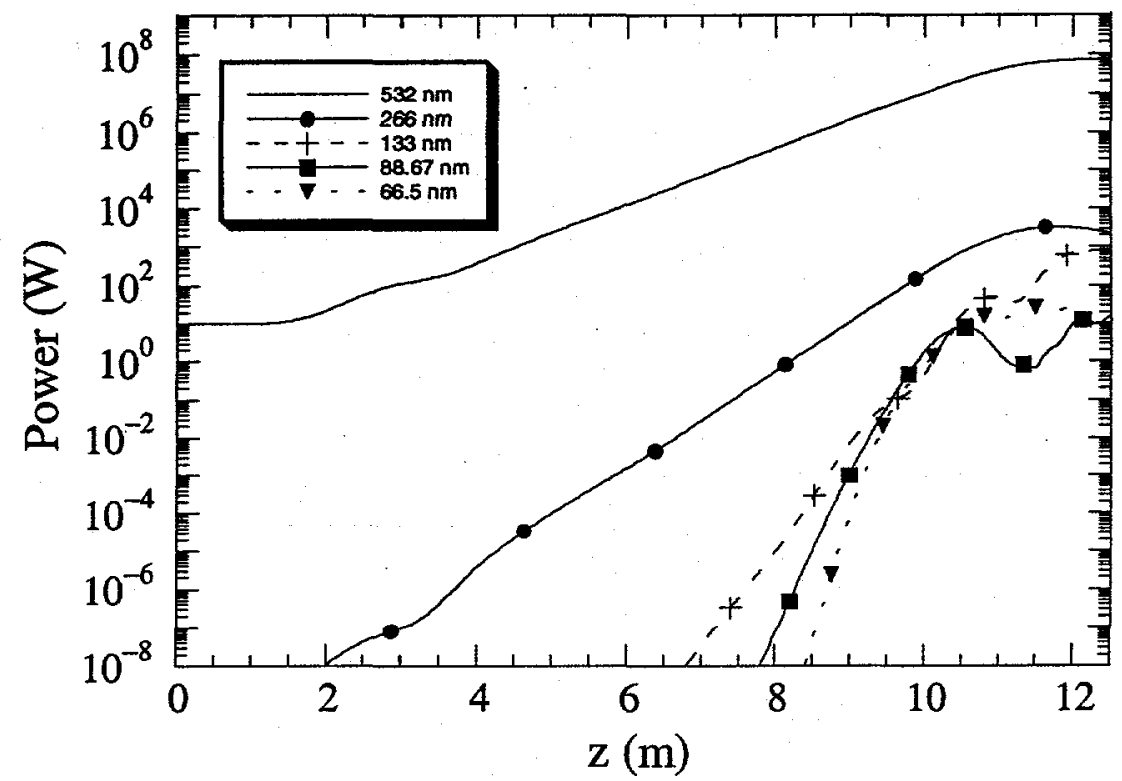

Fig. 2 


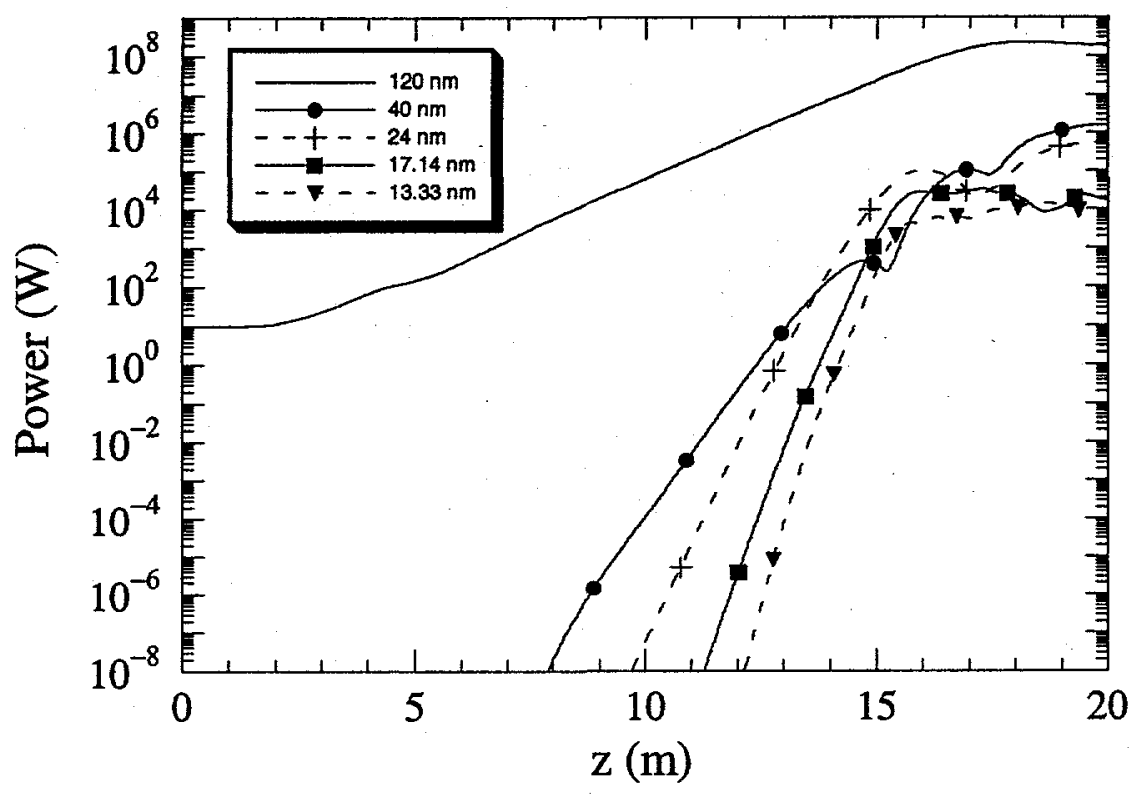

Fig. 3 


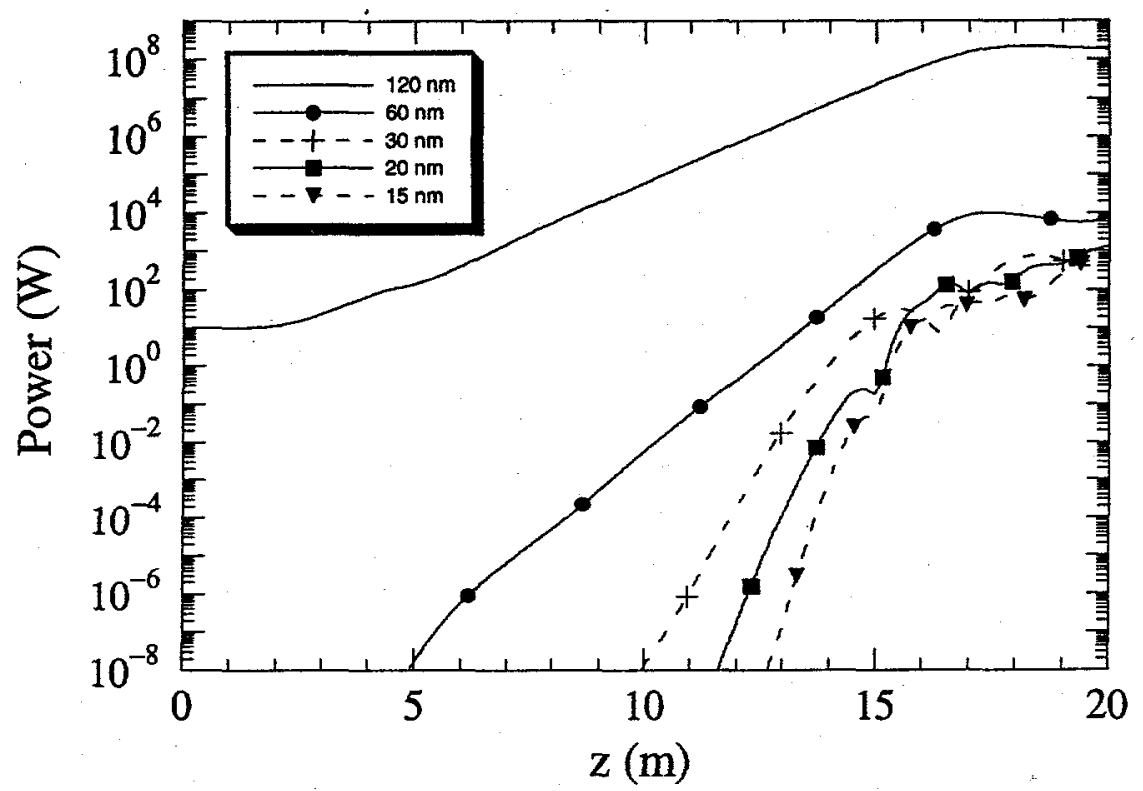

Fig. 4 


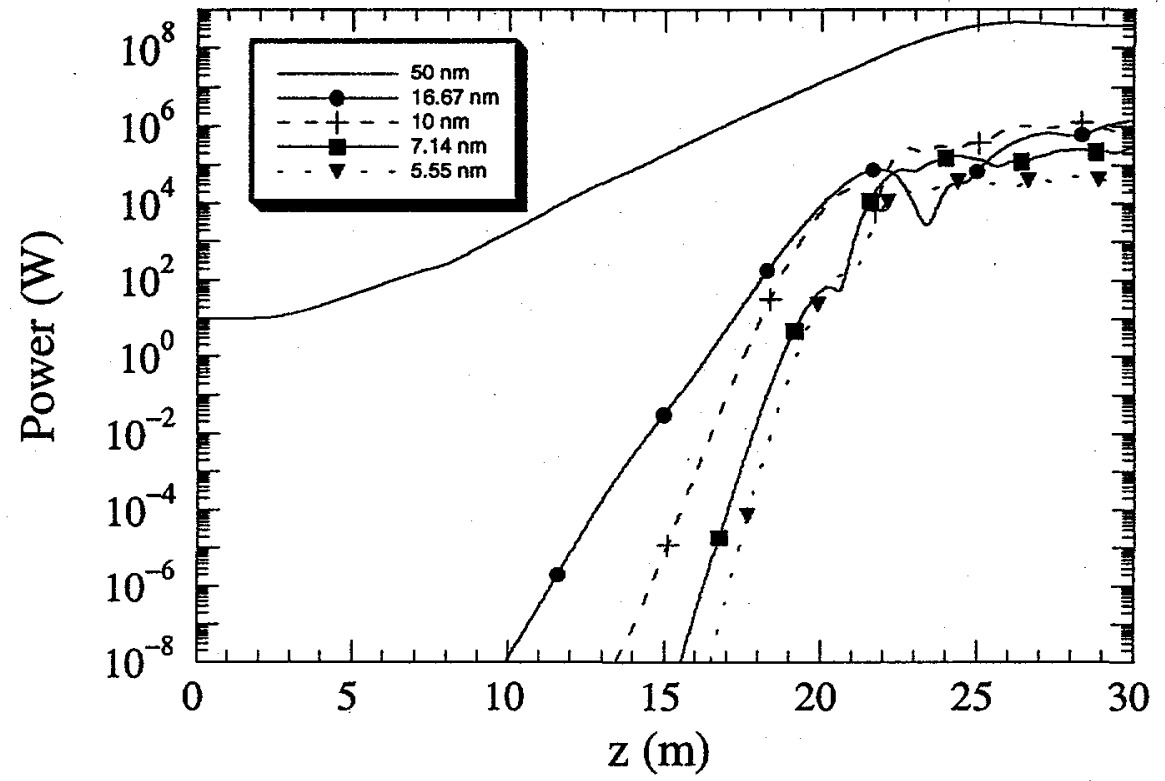

Fig. 5 


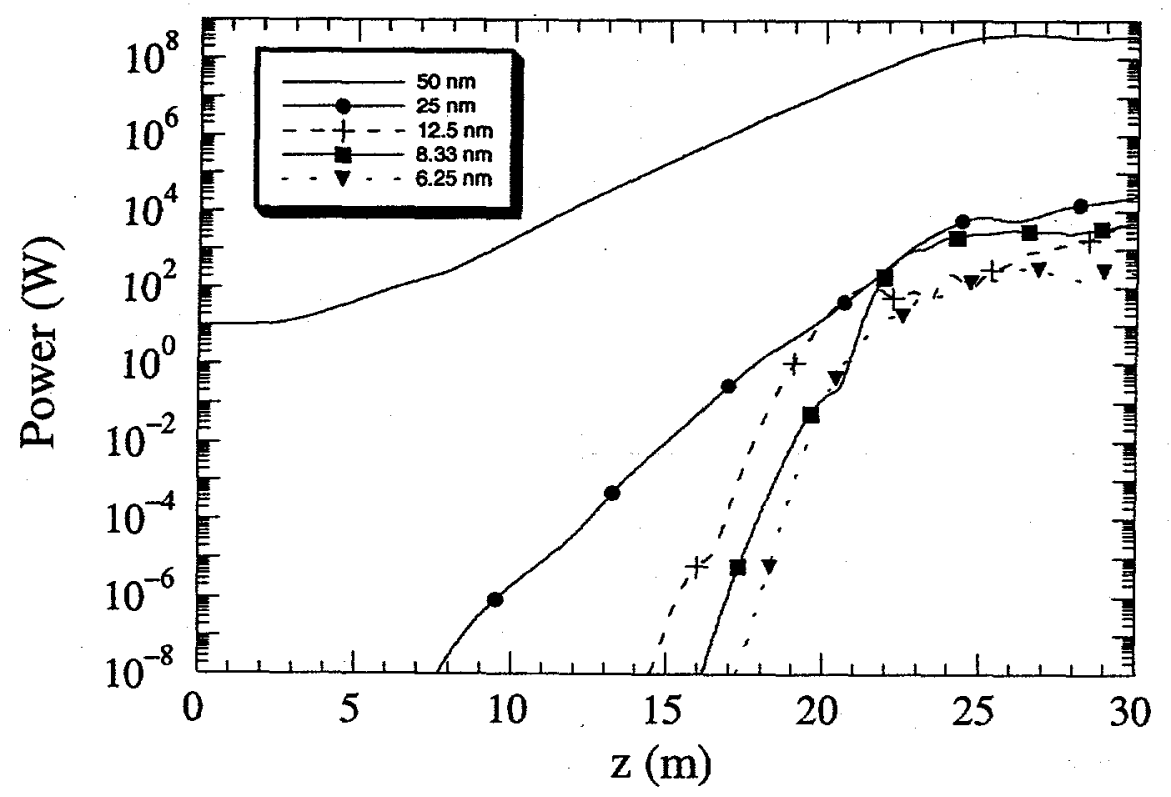

Fig. 6 